\title{
Comment allez-vous ?
}

\section{B. Coffin}

(C) Springer-Verlag France 2011

Le symptôme constipation est particulièrement fréquent, $14 \%$ de la population occidentale en est atteinte [1]. Il est parfois révélateur d'une maladie organique, mais le plus souvent le bilan plus ou moins exhaustif est normal, le diagnostic de « constipation maladie » est alors retenu. Déception de la part du spécialiste ou du généraliste qui a beaucoup de mal à se motiver pour assurer une prise en charge optimale. Et pourtant, le transit digestif est depuis plusieurs siècles une des préoccupations principales de tout sujet adulte.

L'expression « Comment allez-vous ? » que nous utilisons au quotidien pour prendre des nouvelles de chacun signifiait au départ « Comment allez-vous à la selle?».

Comme toute pathologie fréquente, le traitement de la constipation comporte un certain nombre d'idées fausses tant dans le milieu médical que dans la population générale. Ces assertions basées souvent sur des croyances ancestrales, l'auto-intoxication par les matières fécales stagnantes dans le côlon, ou des données médicales anciennes, telles que la toxicité des laxatifs irritants sur les plexus entériques, doivent être combattues pour éviter une prise en charge insuffisante.
Sur le plan thérapeutique, les laxatifs osmotiques restent les traitements de première ligne mais, heureusement, de nouvelles molécules sont en cours de développement, certaines arriveront prochainement sur le marché.

Il est possible que le traitement de cette pathologie chronique se modifie dans les années à venir, permettant une meilleure observance, un soulagement des symptômes satisfaisant et évitant de recourir à des procédures chirurgicales plus ou moins radicales qui ne seront réservées qu'aux patients atteints des formes cliniques les plus sévères, rebelles à tout traitement. On peut donc légitimement espérer que nos patients iront mieux d'ici quelque temps.

\section{Référence}

1. Suares NC, Ford AC (2011) Prevalence of, and risk factors for, chronic idiopathic constipation in the community: systematic review and meta-analysis. Am J Gastroenterol 106:1582-91
B. Coffin $(\bowtie)$

Service d'hépatogastroentérologie, hôpital Louis-Mourier, AP-HP, 178, rue des Renouillers, F-92700 Colombes, France e-mail : benoit.coffin@lmr.aphp.fr

Université Denis-Diderot-Paris-VII, Paris, France 